\title{
Doenças da próstata
}

\section{Prostate diseases}

\section{Miguel Srougi', Leopoldo Alves Ribeiro², Afonso Celso Piovesan ${ }^{2}$, José Roberto Colombo², Adriano Nesrallah ${ }^{2}$}

Srougi M, Ribeiro LA, Piovesan AC, Colombo JR, Nesrallah A. Doenças da próstata. Rev Med (São Paulo). 2008 jul.-set.;87(3):166-77.

\begin{abstract}
RESUMO: As doenças da próstata apresentam grande relevância clínica, pela alta freqüência com que ocorrem no cotidiano e pelas conseqüências que ensejam. A hiperplasia benigna atinge entre 70 e $90 \%$ dos homens maduros, um terço deles apresentam manifestações clinicas mais significativas e, por isto, precisam ser tratados. Nesse sentido, os especialistas dispõe de opções medicamentosas com os inibidores da 5 a-redutase e os bloqueadores $\alpha$-adrenérgicos, ou as intervenções cirúrgicas, reservadas para os pacientes com glândulas de maior dimensão. O câncer da próstata incide em 18\% dos homens, a predisposição aumenta em indivíduos com história familiar ou da raça negra e o diagnóstico da doença é atualmente feito através do toque prostático e de dosagens do PSA, que juntos permitem a identificação da neoplasia em cerca de $80 \%$ dos casos. Nos pacientes com doença localizada o tratamento é feito através de cirurgia radical ou radioterapia. Quando o tumor estendeu-se para os tecidos periprostáticos costumase recorrer à radioterapia e nos casos de tumor disseminado, o processo pode ser controlado com relativa eficiência através da ablação sérica da atividade androgênica.
\end{abstract}

DESCRITORES: Doenças prostáticas. Hiperplasia prostática. Neoplasias da próstata.

\section{INTRODUÇÃO}

$$
A^{p}
$$
pesar da sua pequena dimensão, a próstata costuma ser sede de problemas que tem grande relevância clínica, pela elevada frequência clínica com que ocorrem e por comprometerem a qualidade ou a extensão de vida dos seus portadores. A hiperplasia prostática histológica atinge cerca de $10 \%$ dos homens na década dos 30 anos e é encontrada em $90 \%$ nos indivíduos com mais de 90 anos de idade ${ }^{1,2}$ (Figura 1). Já o câncer da próstata constitui a neoplasia mais frequente dos homens, surgirá em cerca de $18 \%$ dos mesmos até o fim de suas vidas e tem as implicações óbvias negativas sobre a existência desses pacientes.

\footnotetext{
1. Professor Titular, Disciplina de Urologia, Faculdade de Medicina da USP. Chefe da Divisão de Urologia, Hospital das Clínicas da FMUSP.

2. Médico Assistente, Divisão de Urologia, Hospital das Clínicas da FMUSP.

Endereço para Correspondência: Miguel Srougi. Avenida Doutor Enéas de Carvalho Aguiar, 255. São Paulo, SP. Cerqueira César, CEP 05403-900,
} 


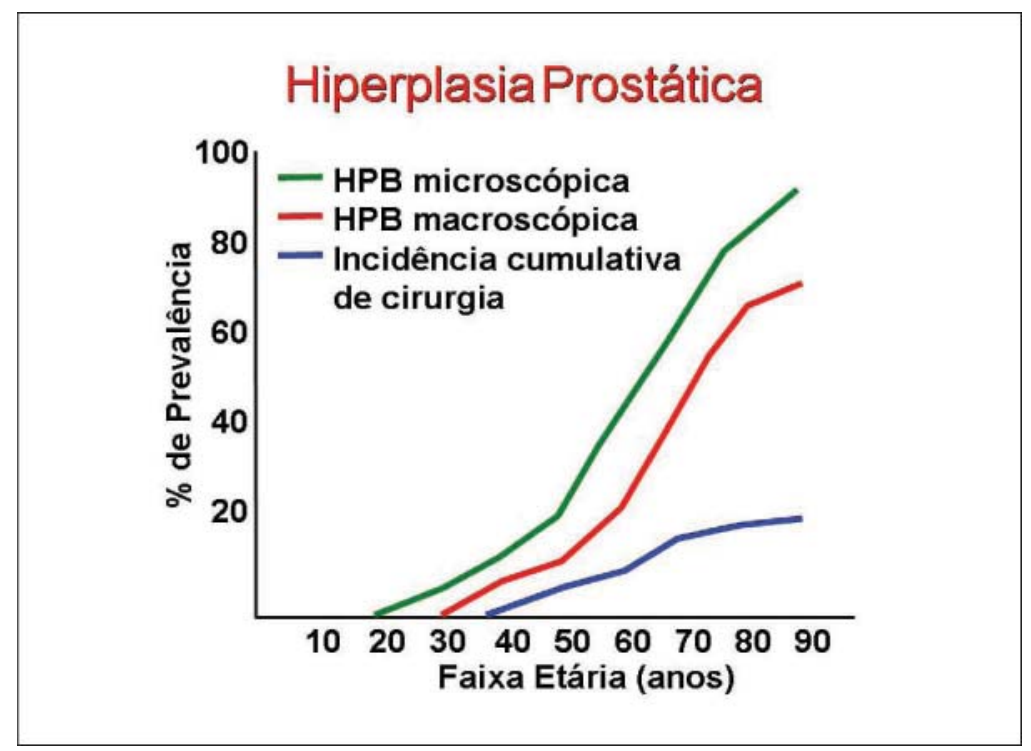

FIGURA 1. Prevalência da hiperplasia benigna da próstata de acordo com diferentes critérios ${ }^{11}$

\section{HIPERPLASIA BENIGNA DA PRÓSTATA}

O aumento da idade e a presença dos testículos representam as determinantes mais importantes para o desenvolvimento da Hiperplasia Benigna da Próstata (HPB). Por outro lado, o papel da hereditariedade parece ser real e filhos de indivíduos com hiperplasia da próstata têm de três a quatro vezes mais chance de serem submetidos à cirurgia prostática por crescimento benigno local ${ }^{1}$.

A história natural dos quadros de HBP é bem conhecida e apresenta algumas implicações práticas relevantes. Os pacientes atingidos pelo processo apresentam sintomas urinários flutuantes, com períodos de exacerbação do quadro e períodos espontâneos de acalmia (Figura 2). Entre 32\% e $70 \%$ dos pacientes com HBP mantidos apenas em observação referem melhora clínica dos sintomas urinários quando seguidos por alguns anos ${ }^{3}$.

\section{Etiopatogenia}

O desenvolvimento da HBP resulta, provavelmente, de vários mecanismos interativos, entre os quais se destacam a ação da diidrotestosterona $(\mathrm{DHT})^{4}$. A testosterona penetra na célula epitelial prostática e por ação de uma enzima, a $5 \alpha$-redutase, é transformada em DHT (Figura 3). Esse intermediário liga-se a receptores androgênicos nucleares, formando um complexo que atua sobre genes específicos, iniciando-se um processo de transcrição e de síntese das proteínas que modulam a proliferação das células epiteliais. A DHT, além de atuar diretamente no contingente celular, representa um potente estimulador das células do estroma prostático, levando-as a secretar, junto com o estradiol, fatores de crescimento que também promovem a proliferação das glândulas prostáticas.

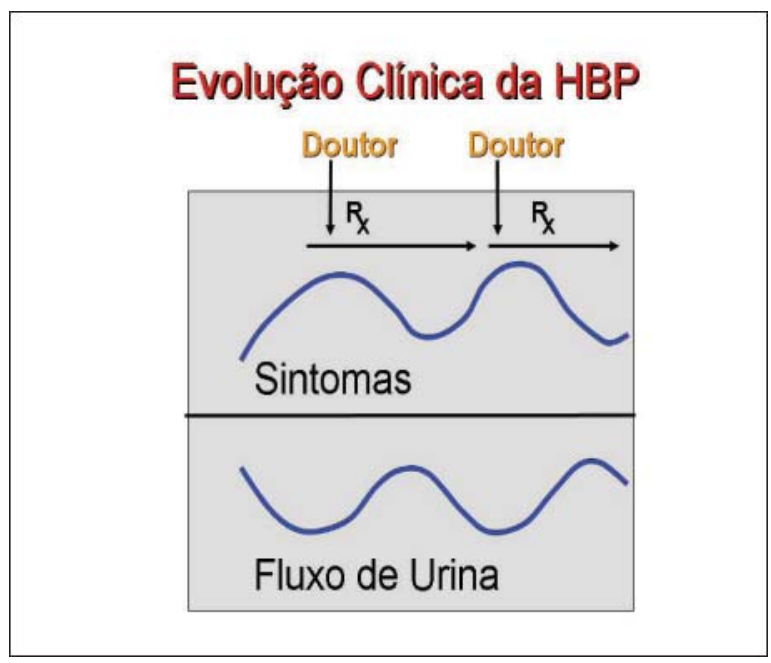

FIGURA 2. História natural da HBP: melhora dos sintomas com o tratamento pode resultar apenas de oscilação casual dos sintomas ${ }^{11}$ 


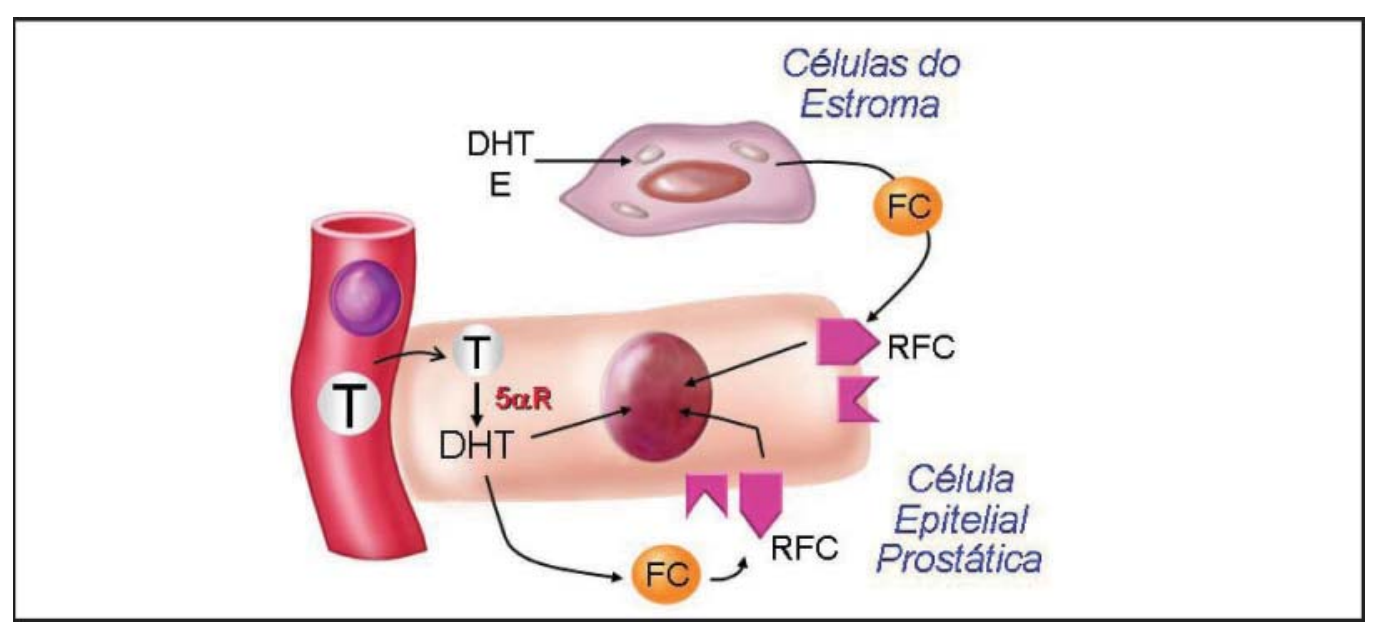

FIGURA 3. Mecanismos endócrinos que regulam a proliferação, atividade e crescimento das células prostáticas ( $\mathrm{T}=$ testosterona; $\mathrm{DHT}=$ dihidrotestosterona; $\mathrm{E}=$ estradiol e estriol; $5 \alpha \mathrm{R}=5$ alfa-redutase; $\mathrm{FC}=$ fatores de crescimento; $\mathrm{RFC}=$ receptores de fatores de crescimento)

Estudos recentes de microarray demonstraram, também, que nos pacientes com hiperplasia prostática existe expressão aumentada de genes relacionados com as reações inflamatórias. Isso faz supor que a gênese da HBP pode estar implicada com reação inflamatória local a agentes nocivos, que incluiriam produtos químicos, proteínas alergênicas ou microorganismos desconhecidos.

\section{Fisiopatologia}

O processo de HBP instala-se na chamada zona transicional da próstata, situada em torno da uretra. $O$ fenômeno de crescimento local condiciona o aparecimento de sintomas miccionais que, na verdade, resultam de três mecanismos fisiopatológicos distintos: 1) obstrução uretral propriamente dita, que produz sintomas obstrutivos; 2) reação do detrusor à obstrução, que leva ao aparecimento de sintomas irritativos; 3 ) estímulos neuronais anormais gerados pela próstata, que também causam sintomas obstrutivos ${ }^{1,5}$

O processo de obstrução uretral decorre do efeito mecânico causado pelo crescimento prostático e de um efeito funcional, relacionado com a contração das fibras musculares existentes no colo vesical, cápsula e estroma prostático. Essas fibras, ricas em receptores $\alpha$-adrenérgicos, tendem a se contrair por estimulação simpática, ocluindo a luz uretral. Esse mecanismo explica os quadros de prostatismo em pacientes com glândulas sem crescimento exagerado.

\section{Manifestações clínicas}

Os pacientes com HBP apresentam mani- festações referidas como "prostatismo", que podem ser divididas em obstrutivas (esforço miccional, hesitância, jato fraco e interrompido, esvaziamento incompleto da bexiga) e irritativas (urgência, polaciúria, nictúria, capacidade vesical reduzida, incontinência de urgência $)^{1,6}$. Essa classificação tem várias implicações práticas. Em primeiro lugar, as intervenções cirúrgicas tendem a se acompanhar de melhores resultados quando predominam as manifestações obstrutivas. Em segundo lugar, quando o quadro irritativo é muito exuberante, deve-se estar atento para a presença de outras doenças causando as manifestações. Incluem-se aqui a bexiga instável do idoso ou relacionada com disfunções neurológicas, infecções locais, litíase vesical e neoplasias de bexiga. Finalmente, quando existem sintomas "obstrutivos" e próstata sem aumento significativo, deve-se cogitar da presença de flacidez vesical neurogênica, como ocorre em alguns pacientes com Diabetes Mellitus, ou de estreitamento uretral.

Pacientes com HBP podem evoluir com complicações como retenção urinária, litíase vesical, infecção urinária, insuficiência renal e hematúria macroscópica ${ }^{1}$. Retenção urinária ocorre em $2 \%$ a $10 \%$ dos casos e está implicada não apenas com falência grave do detrusor, imposta pela obstrução, mas pode, também, estar associada à ingestão de alguns medicamentos (anticolinérgicos, antidepressivos, vasoconstritores nasais) ou à ocorrência de infartos da próstata ou de prostatite aguda. Litíase vesical, que ocorre em $1 \%$ a $4 \%$ dos casos, está quase sempre associada à obstrução prostática e, por isto, tende a recidivar quando se realiza uma intervenção com remoção exclusiva dos cálculos, sem a correção do processo obstrutivo. Infecções urinárias recorrentes surgem em cerca de $5 \%$ dos 
pacientes com HBP, exacerbando os sintomas urinários e, por vezes, desencadeando retenção urinária. Insuficiência renal obstrutiva é observada em $2 \%$ a $3 \%$ dos pacientes com HBP e em metade desses casos o quadro instala-se silenciosamente, o que dificulta o seu diagnóstico. Hematúria macroscópica surge em alguns pacientes com hiperplasia prostática e deve-se à ruptura de vasos submucosos locais. Essa manifestação tende a ceder espontaneamente, mas pacientes nessa situação devem ser explorados cuidadosamente, já que a hematúria pode estar relacionada com a presença de outra afecção, como tumores ou litíase.

\section{Diagnóstico}

Os pacientes com manifestações clínicas da HBP devem ser submetidos a um estudo mínimo que inclui toque digital da próstata, exame neurológico perineal, análise do sedimento urinário e avaliação da função renal por meio de dosagens da creatinina sérica $^{7}$. Medidas do resíduo urinário vesical pósmiccional permitem avaliar a magnitude de falência do músculo detrusor e passam a ter significado quando superam $150 \mathrm{ml}$. Os níveis de PSA sérico elevam-se discretamente nos pacientes com HBP e o exame serve para explorar a presença concomitante de câncer da próstata ${ }^{1}$. Níveis de PSA superiores a $1 / 15$ do peso ou do volume da próstata, calculados pelo estudo de ultra-som, podem indicar a presença de neoplasia maligna local.

O exame de ultra-som abdominal permite avaliar as dimensões da próstata e a presença de anormalidades do trato urinário superior e bexiga, devendo-se ter em mente que esse exame costuma superestimar em até $30 \%$ o volume prostático real.

Medidas do fluxo urinário, por meio de fluxômetros, servem para caracterizar grosseiramente o grau de obstrução uretral. Sob o ponto de vista prático, fluxo urinário máximo maior do que $15 \mathrm{ml} / \mathrm{s}$ é considerado normal e fluxo inferior a $10 \mathrm{ml} / \mathrm{s}$ sugere a existência de processo obstrutivo uretral.

\section{Tratamento}

O tratamento da HBP é feito com o objetivo de aliviar as manifestações clínicas e corrigir as complicações relacionadas com o crescimento prostático. Sob o ponto de vista prático, pacientes com quadros de prostatismo incipiente devem ser apenas acompanhados periodicamente, os casos com sintomas e desconforto moderados devem receber terapêutica medicamentosa e os pacientes com manifestações clínicas exuberantes devem ser tratados cirurgicamente ${ }^{1,6,7}$. Ademais, as intervenções cirúrgicas devem ser realizadas mandatoriamente nos casos de HBP associados à retenção urinária rebelde, hidronefrose e uremia, infecção urinária recorrente, hematúria macroscópica refratária, incontinência urinária paradoxal e litíase vesical.

\section{a) Tratamento farmacológico}

Como foi referido anteriormente, os sintomas da HBP resultam de obstrução uretral mecânica, imposta pelo crescimento prostático, e/ou funcional, relacionada com a contração da musculatura lisa existente ao nível do colo vesical, cápsula e estroma prostático. Em decorrência, os pacientes com obstrução mecânica (próstatas de grande volume) podem ser tratados com medicamentos anti-androgênicos, que promovem atrofia do epitélio glandular, e os casos com oclusão funcional (próstatas de pequeno volume) devem ser tratados com bloqueadores $\alpha$-adrenérgicos, que relaxam a musculatura lisa da próstata e colo vesical.

A finasterida (5 mg uma vez ao dia) e a dutasterida $(0,5 \mathrm{mg}$ uma vez ao dia) constituem os agentes anti-androgênicos mais utilizados em HBP, produzindo uma diminuição de $15 \%$ a $30 \%$ do volume de glândula após seis meses de tratamento. Isso promove um alívio dos sintomas em $30 \%$ a $35 \%$ dos pacientes e esta melhora, em geral, torna-se aparente após três ou quatro meses de tratamento ${ }^{5,8}$. O maior inconveniente desses agentes relaciona-se com o aparecimento de disfunção sexual (queda da libido, da qualidade da ereção e do volume de esperma), que se manifesta em $10 \%$ a $15 \%$ dos casos.

Os bloqueadores $\alpha$-adrenérgicos (doxazocina 4 a $8 \mathrm{mg}$ uma vez ao dia e tamsulocina $0,4 \mathrm{mg}$ uma vez ao dia) acompanham-se de resposta clínica imediata em $60 \%$ dos pacientes, mas não são tão inócuos como os bloqueadores da $5 \alpha$-redutase ${ }^{9}$. Quadros de hipotensão arterial, tontura, congestão nasal e ejaculações secas são observadas em 10\% a 30\% dos pacientes e, às vezes, têm implicações clínicas mais sérias. De modo a reduzir o risco de intercorrências graves, os bloqueadores $\alpha$-adrenérgicos devem ser evitados em pacientes com coronariopatia, acidentes vasculares cerebrais ou insuficiência vascular periférica.

\section{b) Tratamento cirúrgico}

A ressecção transuretral da próstata e a cirurgia abdominal aberta constituem as formas mais eficientes para se tratar pacientes com HBP. Cerca de 90\% dos casos apresentam melhora clínica significativa após essas intervenções e em $10 \%$ dos pacientes podem persistir algumas manifestações urinárias, 
pela existência concomitante de falência vesical, por remoção incompleta da massa prostática obstrutiva ou por seqüelas da cirúrgia, como o aparecimento de estreitamentos uretrais ${ }^{7,8,10}$.

\section{CÂNCER DA PRÓSTATA}

O estudo do câncer de próstata $(\mathrm{CaP})$ reveste-se de grande relevância clínica, em função da sua elevada incidência clínica e das altas taxas de cura desses pacientes quando a doença é detectada em fases iniciais. De todos os tumores malignos detectados em clínica, $29 \%$ originam-se na próstata, numa proporção muito maior que os cânceres de pulmão (15\%) e de cólon (10\%) 2 .

\section{Incidência}

De acordo com estimativa da American Cancer Society, 18\% norte-americanos desenvolverão câncer da próstata em $2008^{2}$ e cerca de $9 \%$ deles irá a óbito pela doença (Figura 4). Se esses números puderem ser transpostos para o nosso país, aproximadamente 144.000 brasileiros serão atingidos pela doença nesse ano e 13.000 morrerão em decorrência.

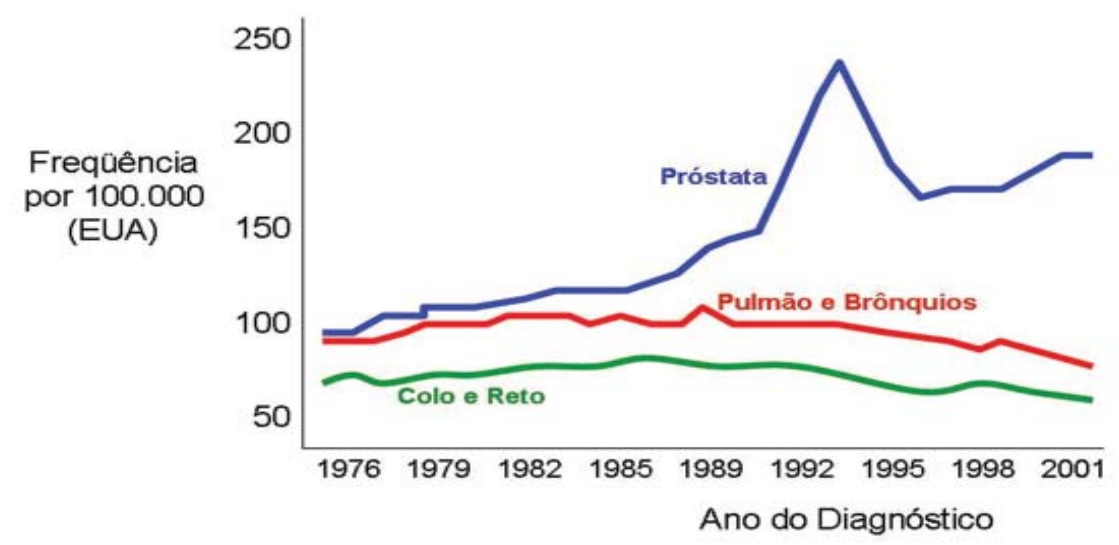

FIGURA 4. Incidência do câncer de próstata verificada nas quatro ultimas décadas nos Estados Unidos

A frequência do câncer da próstata varia geograficamente, com áreas de maior ou menor prevalência. O Canadá, a Jamaica e os países escandinavos apresentam a maior incidência mundial da doença, ao passo que em países do Extremo Oriente a freqüência do problema é 6 a 25 vezes menor ${ }^{11,12}$.

O câncer da próstata apresenta duas características bem peculiares. A sua incidência aumenta com a idade, atingindo quase $50 \%$ dos indivíduos com 80 anos; esse tumor, provavelmente, não poupará nenhum homem que viver até 100 anos (Tabela 1).
Além disso, o câncer da próstata é encontrado em um número elevado de indivíduos, sem lhes causar qualquer mal. Por exemplo, o estudo da próstata em necropsias de homens com idade entre 61 e 70 anos que faleceram sem doença prostática aparente, revela focos neoplásicos em $24 \%$ deles. Contudo, apenas $11 \%$ dos indivíduos dessa faixa etária apresentam, em vida, manifestações clínicas relacionadas com o câncer. Em outras palavras, $13 \%$ dos tumores nesse grupo etário têm um caráter indolente, são assintomáticos e os seus portadores morrem por outros motivos, com o câncer, mas não pelo câncer ${ }^{11}$.

TABELA 1 - Incidência do câncer de próstata em estudos de autópsia e em clínica

\begin{tabular}{lcc}
\hline $\begin{array}{l}\text { Idade } \\
(\text { anos })\end{array}$ & $\begin{array}{r}\text { Câncer em necropsia } \\
(\%)\end{array}$ & $\begin{array}{c}\text { Câncer clínico } \\
(\%)\end{array}$ \\
\hline $50-59$ & 11 & 4 \\
$60-69$ & 24 & 11 \\
$70-79$ & 32 & 23 \\
$>80$ & 44 & 28 \\
\hline
\end{tabular}




\section{Etiologia}

a) Instabilidade genética ${ }^{11}$

Todo homem nasce programado para ter câncer da próstata, pois todos carregam em seu código genético proto-oncogenes, que dão a ordem para uma célula normal se transformar em outra maligna. $O$ câncer da próstata surge porque as múltiplas divisões celulares que vão ocorrendo com o passar dos anos, acompanham-se de discreta fragmentação dos cromossomos com perdas de genes supressores e de ativação de proto-oncogenes, devida a quadros de inflamação ou a influência de mediadores locais (Figura 3).

\section{b) Papel da testosterona ${ }^{11}$}

A regressão da neoplasia após a supressão dos níveis séricos de andrógenos e a observação experimental de que ratos tratados cronicamente com testosterona desenvolvem adenocarcinoma da próstata, fizeram com que se preconizasse uma associação entre câncer da próstata e esses hormônios. Estudos subseqüentes demonstraram que os andrógenos não constituem agentes carcinogenéticos em relação à próstata. Esses hormônios apenas aceleram o crescimento da neoplasia se ela já existir.

\section{Fatores de risco}

a) História familiar

Indivíduos com antecedentes familiares de câncer da próstata têm maior chance de desenvolver a doença. Os riscos aumentam de 2,2 vezes quando um parente de $1^{\circ}$ grau (pai ou irmão) é acometido pelo problema, de 4,9 vezes quando dois parentes de $1^{\circ}$ grau são portadores do tumor e de 10,9 vezes quando três parentes de $1^{\circ}$ grau têm a doença. Nos casos hereditários, o câncer manifesta-se mais precocemente, freqüentemente antes dos 50 anos $^{11}$.

\section{b) Raça}

A incidência do CaP é cerca de $70 \%$ maior em negros e $70 \%$ menor em orientais e índios, quando comparada com a freqüência em brancos. Ademais, a mortalidade pela doença é cerca de três vezes maior em negros, contribuindo para isto, não apenas fatores genéticos, mas também menor acesso aos cuidados de saúde ${ }^{11}$.

\section{Patologia}

Cerca de $98 \%$ das neoplasias da próstata são representadas pelos adenocarcinomas e o restante compreende casos de sarcomas, carcinoma epidermóide e carcinoma de células transicionais ${ }^{11}$.
Os adenocarcinomas localizam-se na zona periférica da glândula em cerca de $75 \%$ dos casos, na zona transicional em aproximadamente $25 \%$ dos pacientes e na zona central em menos de $5 \%$ dos casos.

A diferenciação glandular nos casos de adenocarcinoma da próstata constitui um importante fator prognóstico, relacionando-se com o comportamento biológico do tumor e a sobrevida do paciente. Sob o ponto de vista prático, o sistema de graduação histológico mais utilizado é o proposto por Gleason, que valoriza principalmente o padrão glandular e a relação entre as glândulas e o estroma prostático. Nesse sistema, os tumores são classificados em cinco graus, denominando-se grau 1 as lesões mais diferenciadas e grau 5 as mais indiferenciadas. Como os adenocarcinomas da próstata apresentam mais de um padrão histológico, o diagnóstico final na escala de Gleason é dado pela soma dos graus do padrão primário (predominante) e do padrão secundário (segunda menor área representada), o que faz com que as neoplasias mais diferenciadas sejam classificadas como escore $2(1+1)$ e as mais anaplásicas sejam caracterizadas por escore $10(5+5)$. Como mostra a Figura 5, a sobrevida dos pacientes com câncer da próstata relaciona-se diretamente com o escore histológico pelo sistema de Gleason.

\section{Estagiamento}

A evolução dos pacientes com adenocarcinoma da próstata está intimamente relacionada com a extensão da neoplasia e, por isto, União Internacional Contra o Câncer (UICC) propôs a utilização do sistema TNM em câncer de proposta, de modo a padronizar a classificação dos pacientes com a doença e permitir estudos comparativos mais precisos (Quadro 1).

\section{História natural}

A evolução dos pacientes com câncer da próstata é relativamente imprevisível, com casos de rápida disseminação da neoplasia, antes mesmo de surgirem sintomas locais, e casos de evolução lenta e indolente, com lesões que permanecem estacionárias ${ }^{11}$. Esse fenômeno está refletido na Tabela 2. Quando pacientes com CaP são acompanhados sem tratamento por períodos longos de tempo, muitos acabam necessitando de uma intervenção terapêutica por sinais de progressão da doença. Contudo, entre $26 \%$ e $57 \%$ dos casos mantém-se com o quadro inicial estável e permanecem livres de tratamento até sete anos após o diagnóstico. 

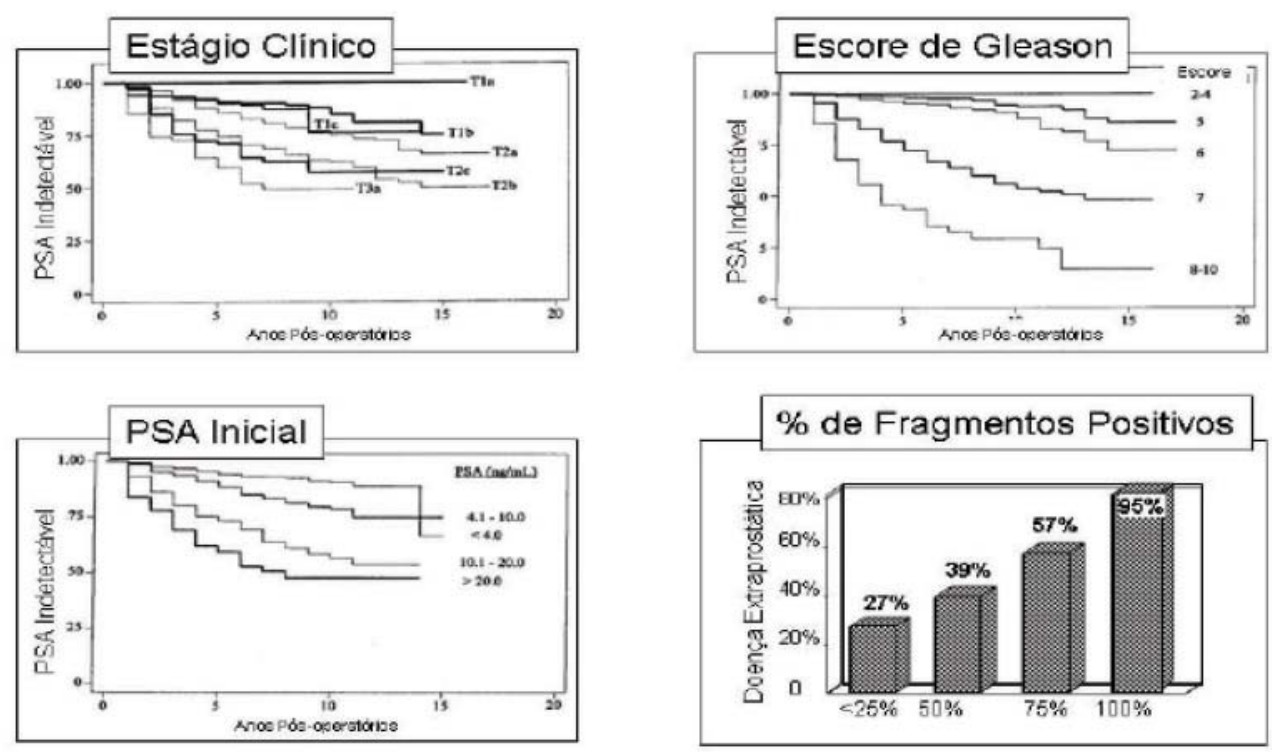

FIGURA 5. Sobrevida dos pacientes com câncer da próstata em função do estágio clinico da doença, escore histológico de Gleason e PSA sérico inicial. A porcentagem de fragmentos positivos relaciona-se com a presença de doença extraprostática

QUADRO 1. Estagiamento do câncer de próstata

\begin{tabular}{|c|c|c|}
\hline TNM & Whitmore & Definição \\
\hline$T_{0}$ & & Sem evidência de tumor prostático \\
\hline $\mathrm{T}_{1 \mathrm{a}}$ & $A_{1}$ & Tumor năo-palpável, $<5 \%$ \\
\hline $\mathrm{T}_{1 \mathrm{~b}}$ & $A_{2}$ & Tumor não-palpável, >5\% \\
\hline$T_{10}$ & - & Tumor năo-palpável, PSA alterado \\
\hline $\mathrm{T}_{2 \mathrm{a}}$ & $\mathrm{B}_{1}$ & Nódulo $<1 / 2$ lobo \\
\hline $\mathrm{T}_{2 \mathrm{~b}}$ & \multirow{2}{*}{$\mathrm{B}_{2}$} & Nódulo $>1 / 2$ lobo \\
\hline $\mathrm{T}_{20}$ & & Nódulo bilateral \\
\hline $\mathrm{T}_{3 \mathrm{a}}$ & $C_{1}$ & Extensão periprostática mínima \\
\hline $\mathrm{T}_{3 \mathrm{~b}}$ & \multirow{2}{*}{\}$C_{2}$} & Invasåo do colo vesical \\
\hline$T_{30}$ & & Invasão das vesículas seminais \\
\hline $\mathrm{T}_{4}$ & - & Invasão da parede pélvica \\
\hline $\mathrm{N}_{0}$ & & Sem metástases em linfonodos \\
\hline$N_{1}$ & \multirow{2}{*}{\}$D_{1}$} & Metástases em linfonodos iliacos \\
\hline $\mathrm{N}_{2}$ & & Metástases em linfonodos aórticos \\
\hline $\mathrm{N}_{3}$ & - & Metástases em linfonodos aórticos \\
\hline$M_{0}$ & - & Sem metástases sistêmicas \\
\hline$M_{1}$ & $\mathrm{D}_{2}$ & Metástases sistêmicas \\
\hline
\end{tabular}


Srougi M, Ribeiro LA, Piovesan AC, Colombo JR, Nesrallah A. Doenças da próstata.

TABELA 2. História natural dos casos não-tratados de câncer localizado da próstata

\begin{tabular}{|c|c|c|c|c|}
\hline \multirow[t]{2}{*}{ Autores } & \multirow[t]{2}{*}{ № } & \multicolumn{2}{|c|}{5 anos $(\%)$} & \multirow[b]{2}{*}{ Óbitos } \\
\hline & & Progressão & Metástases & \\
\hline Georges, 1988 & 120 & 84 & 12 & 20 \\
\hline Johansson, 1989 & 223 & 31 & 9 & 8 \\
\hline Whitmore, 1991 & 75 & 69 & 37 & 0 \\
\hline Adolfsson, 1992 & 122 & 55 & 14 & 1 \\
\hline
\end{tabular}

Vários parâmetros podem ser utilizados para definir o prognóstico de pacientes com adenocarcinoma de próstata ${ }^{11,13}$. O estágio inicial do tumor, o escore histológico, o volume da neoplasia, as medidas de PSA e o número de fragmentos de biópsia envolvidos representam os principais métodos de previsão prognóstica desses casos ${ }^{11}$. Os pacientes com estágios $T_{1}$ e $T_{2}$ apresentam elevadas chances de cura, enquanto aqueles com estágio $\mathrm{M}+$ tendem a evoluir de forma precária. Da mesma forma, os pacientes com tumores bem diferenciados (escores 2 a 6) quase sempre são curadas da doença quando tratados adequadamente enquanto a maioria dos casos de neoplasias indiferenciadas (escores 8 a 10) evoluem de forma mais precária e imprevisível. $\mathrm{O}$ volume tumoral também esta implicado com o prognóstico dos casos de câncer da próstata, não se observando metástases ósseas ou em linfonodos ilíacos nos tumores prostáticos com menos de 3 centímetros cúbicos de volume e a ocorrência de tais fenômenos em mais de $80 \%$ dos tumores com mais de $12 \mathrm{cc}$ de volume. Os níveis séricos de PSA elevam-se progressivamente à medida que aumenta a extensão e o estágio de neoplasia. Pacientes com tumores localizados costumam evidenciar níveis séricos inferiores a $20 \mathrm{ng} / \mathrm{ml}$, e nos casos de doença regional extra-prostática esses níveis costumam se situar entre 20 e $80 \mathrm{ng} / \mathrm{ml}$ e quando a neoplasia se dissemina os valores de PSA ultrapassam 100ng/ $\mathrm{ml}$. A extensão da doença e a sua agressividade relacionam-se diretamente com a porcentagem de fragmentos positivos na biópsia. Pacientes com mais de que $50 \%$ dos fragmentos positivos apresentam chances elevadas de portar extensão extra-prostática da neoplasia e, portanto, doença mais grave.

\section{Clínica}

No passado, a maioria dos pacientes com câncer da próstata apresentava-se com neoplasia disseminada, mas, em decorrência dos programas de detecção precoce e orientação preventiva, esse fenômeno se modificou e, atualmente, a maior parte desses casos é identificada ainda com doença localizada $^{11}$. Atualmente entre $88 \%$ e $92 \%$ dos novos casos evidenciam neoplasia confinada à glândula $e$ apenas $4 \%$ a $6 \%$ dos pacientes apresentam-se com tumor metastático.

Nos pacientes com tumor circunscrito à próstata, a doença é assintomática, Por outro lado, mais de $90 \%$ dos pacientes com adenocarcinoma da próstata localmente avançado apresentam-se com manifestações de obstrução infravesical e evidenciam hematúria macroscópica, em geral relacionada com infiltração do trígono pelo tumor ${ }^{11}$. O aparecimento súbito de obstrução infravesical em um paciente com padrão miccional recente satisfatório é manifestação comum em câncer de próstata. Em casos de hiperplasia benigna, os sintomas obstrutivos tendem a evoluir de forma mais lenta.

De forma incomum, pacientes com câncer de próstata podem apresentar dores ósseas, uremia, anemia, perda de peso, adenopatia cervical ou inguinal, linfedema, trombose venosa de membros inferiores ou hemospermia, como primeira manifestação da doença.

\section{Diagnóstico}

a) Detecção do tumor primário

A detecção do câncer da próstata é feita pelo toque digital da glândula, através de medidas do PSA sérico e da ultra-sonografia transretal. $O$ toque digital tem sensibilidade que varia entre $18 \%$ e 35\%, já as dosagens do PSA têm sensibilidade um pouco maior que o toque digital, da ordem de $40 \%$ a $50 \%{ }^{11}$. Sob o ponto de vista prático, níveis séricos de PSA inferiores a $2,5 \mathrm{ng} / \mathrm{ml}$, em pacientes com o toque prostático normal, acompanham-se de riscos desprezíveis de presença de câncer na próstata, o que permite que esses casos sejam apenas seguidos clinicamente ${ }^{14}$. Por outro lado, níveis superiores a $2,5 \mathrm{ng} / \mathrm{ml}$ acompanham-se de riscos substanciais da doença, que são da ordem de $35-40 \%$ quando o PSA está entre 2,5 e 10. Quando os níveis de PSA situamse entre 10 e $20 \mathrm{ng} / \mathrm{ml}$, a chance de existir neoplasia prostática é de cerca de $55 \%$. Por isso, biopsia da próstata deve ser sempre indicada quando os níveis de PSA ultrapassam $2,5 \mathrm{ng} / \mathrm{ml}$ e não existem outras explicações para tal elevação. 
b) Biópsia da próstata

A biópsia da próstata deve ser indicada em todos os pacientes com áreas de maior consistência na glândula e/ou com elevação dos níveis séricos de PSA. Essas alterações traduzem a presença de adenocarcinoma em $5 \%$ a $95 \%$ dos casos, sendo que afecções benignas, como nódulos de hiperplasia benigna, infecção, cálculos ou infartos prostáticos, podem ser responsáveis por alterações encontradas no toque retal ou nas medidas de PSA e simular a presença de câncer local ${ }^{11}$.

A realização de biópsia prostática com ajuda da USTR e com sedação endovenosa, pode se acompanhar de resultados falso-negativos em 10\% a $22 \%$ dos casos. Isso exige que, nos pacientes com indícios significativos de câncer e biópsia negativa, o procedimento seja repetido precocemente.

\section{Estagiamento}

O estagiamento clínico dos pacientes com câncer da próstata é fundamental para o planejamento terapêutico desses $\operatorname{casos}^{11}$. Além do toque retal, que permite avaliar localmente a extensão do tumor, o estagiamento é feito por meio de medidas das fosfatases ácida e alcalina, dosagem do antígeno prostático especifico, cintilografia óssea, ultra-sonografia transretal, estudo de ressonância magnética da pelve e do retroperitônio, PET-CT e linfadenectomia ilíaca.

\section{Tratamento}

Ao se planejar o tratamento dos casos de câncer da próstata, deve-se levar em consideração a extensão da doença, o grau histológico do tumor e as condições gerais do paciente ${ }^{15}$. Os tumores localizados inteiramente dentro da glândula (estágio $T_{1} e$ $\mathrm{T}_{2}$ ) nem sempre precisam ser tratados, mas, quando isto for necessário, pode-se recorrer à cirurgia, à radioterapia conformacional ou à braquiterapia com sementes de iodo radioativo. Quando o câncer atinge os envoltórios da próstata (estágio $T_{3}$ ) costuma-se indicar tratamento radioterápico conformacional associado à terapêutica hormonal anti-androgênica. Finalmente, quando tumor se estende para outros órgãos (estágios $\mathrm{N}+$ e/ou $\mathrm{M}+$ ) a doença é tratada com castração ou hormônios anti-androgênicos.

O grau histológico do tumor também deve ser levado em conta quando se planeja o tratamento dos casos de câncer da próstata, uma vez que as lesões bem diferenciadas (escore de Gleason 2-6) tendem a ter um comportamento mais indolente e, por isto, podem ser tratadas de forma mais conservadora. Por outro lado, as neoplasias indiferenciadas (escores de Gleason 8-10), são extremamente agressivas e respondem de forma imprevisível às diferentes opções de tratamento. Isto justifica a adoção de terapia combinada nesses casos, em geral, a associação de cirurgia ou radioterapia com terapêutica hormonal.

Finalmente, a estratégia de tratamento dos casos de câncer da próstata deve levar em conta as perspectivas de vida do paciente. A orientação conservadora (vigilância clínica ou tratamento hormonal) está justificada nos casos com perspectiva de vida menor que 10 anos, quer pela idade avançada do paciente, quer pela existência de doenças complexas associadas. Quando as condições gerais e a idade prenunciam chances razoáveis de sobrevida de mais de 10 anos, o tratamento curativo radical deve ser adotado.

Entre $10 \%$ e $15 \%$ dos pacientes com CaP apresentam lesões com características muito favoráveis e que, em conseqüência, evoluem de forma muito lenta, com pequeno risco de gerar metásta$\operatorname{ses}^{11}$. Muitos desses casos, principalmente quando a idade do paciente é mais avançada, a doença não precisa ser tratada. Cabe enfatizar que a opção do não-tratamento deve ser adotada com cautela, em pacientes motivados e acompanhados cuidadosamente. A adoção desta estratégia em neoplasias mais agressivas pode transformar uma doença curável em outra não-curável.

\section{a) Tratamento do câncer localizado}

Uma grande controvérsia envolve o tratamento dos pacientes com câncer localizado da próstata ${ }^{11,15}$. Cirurgiões e radioterapeutas proclamam que a cirurgia radical e a radioterapia conformada, respectivamente, representam a maneira ideal de se tratar tais casos, referindo índices de sobrevida de 10 anos entre $60 \%$ e $95 \%$. Contudo, de acordo com os dados mais recentes, entre $89 \%$ e $93 \%$ dos pacientes submetidos à prostatectomia radical e entre $60 \%$ e $86 \%$ dos casos tratados com radioterapia externa ou braquiterapia estão curados após 10 anos de acompanhamento ${ }^{11,15,16}$.

Embora o valor curativo da prostatectomia radical seja inquestionável e a intervenção é atualmente realizada com baixa morbidade, ela pode provocar impotência sexual e incontinência urinária, comprometendo a qualidade de vida do paciente. A impotência, que se caracteriza por perda das ereções penianas, surge em $95 \%$ dos casos operados com mais de 70 anos de idade, em $50 \%$ dos indivíduos com 55 a 65 anos e em $15 \%$ a $20 \%$ dos pacientes com menos de 55 anos. Incontinência urinária moderada ou grave em $3 \%$ a $5 \%$ dos casos quando a intervenção é realizada por equipes habilitadas.

A radioterapia conformada, apesar da sua 
característica não-invasiva, também se acompanha de efeitos indesejáveis. Entre $40 \%$ e $50 \%$ dos pacientes desenvolvem impotência sexual, que surge um ou dois anos após o tratamento e, por isto, nem sempre é atribuída à radioterapia. Além disto, cerca de $20 \%$ apresentam reação actínica em reto, ânus e bexiga durante o tratamento, que tende a melhorar após um ou dois meses, mas pode se perpetuar em cerca de $10 \%$ dos pacientes.

b) Tratamento do câncer localmente avançado

Define-se por câncer localmente avançado aquelas neoplasias que apresentam, ao toque ou por estudos de imagem, extensão da doença para os tecidos periprostáticos, mas sem metástases aparentes. Incluem-se também nesse grupo, os pacientes com níveis de PSA superiores a $20 \mathrm{ng} / \mathrm{ml}$ e os casos com escore de Gleason 9-10 ou $100 \%$ dos fragmentos da biópsia positivas para tumor, nos quais as chances de envolvimento periprostático pela neoplasia são elevadas.

De acordo com dados recentes da literatu$\mathrm{ra}^{15}$, esses pacientes são melhor controlados com o emprego de hormonioterapia anti-androgênica por três anos, associado à radioterapia externa iniciada no $3^{\circ}$ ou $4^{\circ}$ mês de tratamento. Outra opção que vem sendo proposta para esses casos é o da realização de cirurgia radical, associada a radioterapia externa local no pós-operatório, com resultados recentes promissores.

c) Tratamento do câncer disseminado

Apesar de sucessivas tentativas de se controlar a doença disseminada por outros meios, a terapêutica endócrina representa, ainda, o único método eficiente e objetivo para se deter a evolução do câncer da próstata em suas fases mais avançadas. A testosterona estimula a função e a proliferação das células prostáticas, de modo que intervenções que reduzem os seus níveis séricos inibem a divisão das mesmas e produzem regressão tumoral.

Sob o ponto de vista clínico, a supressão da atividade androgênica pode ser realizada de diferentes formas (Figura 6): a) orquiectomia bilateral; b) supressão da liberação hipotalâmica ou hipofisária de $\mathrm{LH}$ e FSH, através de, respectivamente estrógenos ou análogos do LHRH; c) bloqueio da ação periférica da testosterona através dos anti-androgênicos; d) bloqueio da síntese de testosterona pela ciproterona ${ }^{11,17}$. Embora se atribua a estas modalidades a mesma eficiência terapêutica, a vivência clínica tem indicado que a orquiectomia e a estrogenoterapia representam as alternativas mais eficazes nesses casos. Os análogos do LHRH apresentam uma eficiência um pouco menor e os anti-androgênicos periféricos são os menos atuantes sob o ponto de vista clínico.

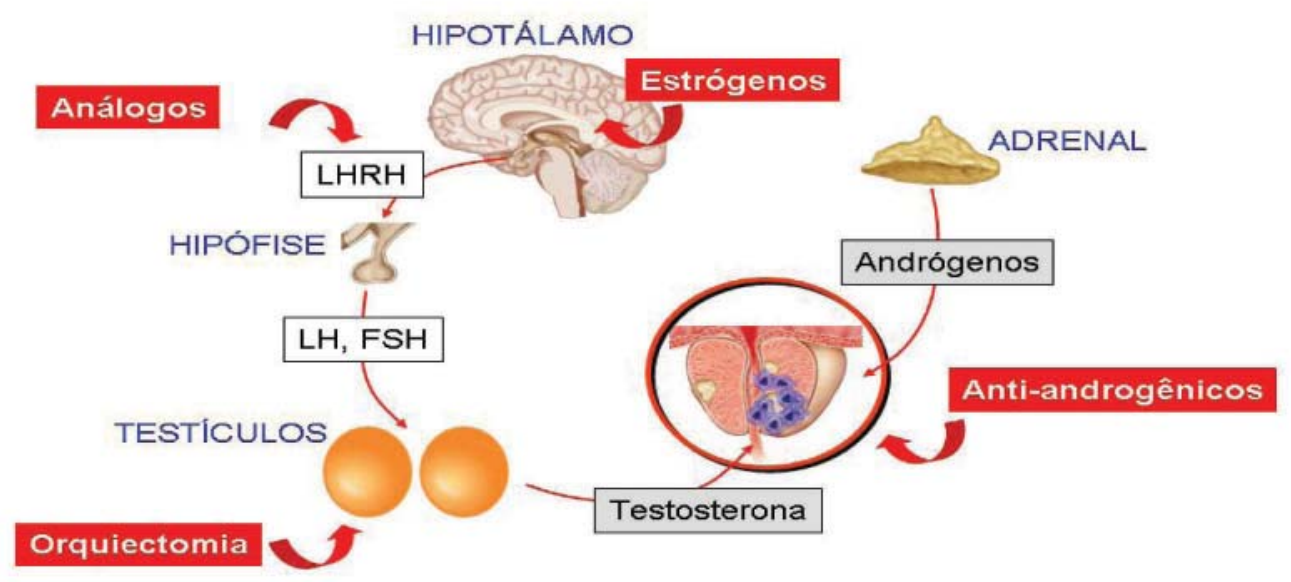

FIGURA 6. Formas de ablação da atividade androgênica do plasma

Como esses métodos apresentam efeitos colaterais e inconvenientes de maior significado, o tratamento dos pacientes com CaPD deve ser personalizado ${ }^{11,17}$. Dessa forma, pacientes institucio- nalizados, com recursos financeiros limitados e com dificuldade de acesso a cuidados médicos, são mais bem tratados com a orquiectomia, que os protege de forma definitiva. Nos pacientes sem afecções 
cardiovasculares e que não aceitam a castração, pode-se recorrer aos estrógenos, como por exemplo, o dietilstilbestrol, 1 a $3 \mathrm{mg}$ ao dia. Nos pacientes com maior disponibilidade econômica, a terapêutica antiandrogênica pode ser realizada com os análogos do LHRH, que além de não induzirem ginecomastia ou complicações cardiovasculares, são de fácil administração, feita através de injeções repetidas a cada 1, 2 ou 3 meses. A utilização isolada dos antiandrogênicos periféricos representa a modalidade menos atuante em casos de CaPD, mas, sob o ponto de vista clínico, o emprego desses agentes está indicado em pacientes que desejam preservar a função sexual. Realmente, a flutamida (750mg ao dia), a nilutamida (150mg ao dia) e a bicalutamida (50mg ao dia) permitem que cerca de $50 \%$ dos pacientes tratados mantenham-se sexualmente ativos, já que os níveis séricos de testosterona permanecem elevados durante a administração dessas medicações ${ }^{11,18}$.

\section{d) Tratamento do câncer hormônio-refratário}

A grande maioria das neoplasias malignas da próstata responde, de início, à ablação androgênica, mas com o tempo esses tumores adquirem autonomia e passam a se proliferar mesmo na ausência de testosterona.

A emergência de clones celulares resistentes ao tratamento anti-androgênico explica os índices precários de respostas quando terapêutica endócrina de segunda linha é introduzida em pacientes que falharam ao tratamento primário. Por isso, novas estratégias, como bloqueio adrenal (glicocorticóides ou cetoconazol), terapêutica citotóxica combinada (docetaxel, mitoxantrona, ciclofosfamida), inibição dos fatores de crescimento e terapia genética, vem sendo exploradas clinicamente ${ }^{11,18}$.

\section{Prevenção}

A prevenção do câncer da próstata não pode ser feita de forma eficiente, porque ainda não são conhecidos os fatores que modificam a maquinaria celular, tornando-a maligna ${ }^{11}$. Hábitos dietéticos específicos talvez possam reduzir os riscos de câncer da próstata. Nesse sentido, tem-se recomendado alimentação com baixo teor de gordura animal, hábito comum nos países onde a incidência da doença é baixa (o ideal é que sejam ingeridos diariamente apenas $15 \%$ do total de calorias sob forma de gordura). O consumo abundante de tomate cozido e seus derivados parece diminuir em $35 \%$ os riscos de câncer da próstata. $O$ efeito benéfico do tomate resultaria da presença de grandes quantidades de licopeno, um betacaroteno natural precursor da vitamina A. Finalmente, complementação dietética com vitamina $E$ ( $800 \mathrm{mg}$ ao dia) e com selênio (200 $\mu \mathrm{g}$ ao dia) talvez tenha um efeito protetor contra o câncer da próstata, reduzindo riscos de ocorrência da doença em $32 \%$ a $63 \%$ dos pacientes assim tratados.

Srougi M, Ribeiro LA, Piovesan AC, Colombo JR, Nesrallah A. Prostate diseases. Rev Med (São Paulo). 2008 jul.-set.;87(3):166-77.

\begin{abstract}
Prostate diseases occur with high frequency in the clinical setting and can compromise patients' quality or extent of life. Benign prostatic hyperplasia is seen in $70-90 \%$ of men and is the cause of disturbing urinary symptoms in one third of them. In these symptomatic patients medical treatment with $5 \alpha$-redutase inhibitors or $\alpha$-blockers can improve the urinary manifestations. When obstructive urinary symptoms are prominent surgical treatment can successfully improve patient's outcome. Prostate cancer will affect $18 \%$ of adult men, familiar history and black men are more susceptible to the disease and precise detection of the tumor can be done with serum PSA measurements and digital rectal exam. Patients with confined disease are usually treated with radical surgery or radiotherapy, locally advanced tumors are best managed with external radiotherapy and metastatic disease can be improved with androgen suppressive therapy.
\end{abstract}

KEY WORDS: Prostatic diseases. Prostatic hyperplasia. Prostatic neoplasms.

\title{
REFERÊNCIAS
}

1. Srougi M. Hiperplasia prostática. Rio de Janeiro: Record; 1995.

2. Jemal A, Siegel R, Ward E, Murray T, Xu J, Thun MJ. Cancer statistics 2007. CA Cancer J Clin.
2007;57:43-66.

3. Fitzpatrick JM. The natural history of benign prostatic hyperplasia. BJU Int. 2006;97(Suppl 2):3-6.

4. Eaton CL. Aetiology and pathogenesis of benign 
prostatic hyperplasia. Curr Opin Urol. 2003;13:7-10.

5. Andriole G, Bruchovsky N, Chung LW, Matsumoto AM, Rittmaster R, Roehrborn C, Russell D, Tindall D. Dihydrotestosterone and the prostate: the scientific rationale for 5alpha-reductase inhibitors in the treatment of benign prostatic hyperplasia. J Urol. 2004;172:1399-403.

6. Barry MJ. Evaluation of symptoms and quality of life in men with benign prostatic hyperplasia. Urology. 2001;58(Suppl 1):25-32.

7. Bhargava S, Canda AE, Chapple CR. A rational approach to benign prostatic hyperplasia evaluation: recent advances. Curr Opin Urol. 2004;14:1-6.

8. McNaughton-Collins M, Barry MJ. Managing patients with lower urinary tract symptoms suggestive of benign prostatic hyperplasia. Am J Med. 2005;118:331-9.

9. Garg G, Singh D, Saraf S, Saraf S. Management of benign prostate hyperplasia: an overview of alpha-adrenergic antagonist. Biol Pharm Bull. 2006;29:554-8.

10. Reich O, Gratzke C, Stief CG. Techniques and longterm results of surgical procedures for $\mathrm{BPH}$. Eur Urol. 2006;49:970-8.

11. Srougi M. Cancer da próstata. In: Srougi M, Simon SD, editores. Cancer urológico. 2a ed. São Paulo: Platina; 1995. p. 281-359.

12. Shimizu H, Ross KR, Bernstein L, Yatani R, Hender- son BE, Mack TM. Cancers of the prostate and breast among Japanese and immigrants in Los Angeles County. Br J Cancer. 1991;63:963-66.

13. Crippa A, Srougi M, Dall'Oglio MF, Antunes AA, Leite $K R$, Nesrallah LJ, Ortiz V. A new nomogram to predict pathologic outcome following radical prostatectomy. Int Braz J Urol. 2006;32:155-64.

14. Thompson IM, Pauler DK, Goodman PJ, Tangen CM, Lucia MS, Parnes HL, et al. Prevalence of prostate cancer among men with a prostate-specific antigen level of 4.0ng per milliliter. N Eng J Med. 2004;350:2239-46

15. Middleton RG, Thompsom IM, Austenfeld MS, Cooner WH, Gibbons RJCRP, Miller HC, et al. Prostate cancer clinical guidelines panel summarys report on the management of clinically localized prostate cancer. J Urol. 1995;154:2144-8.

16. Berglund RK, Jones JS, Ulchaker JC, Fergany A, Gill I, Kaouk J, et al. Radical prostatectomy as primary treatment modality for locally advanced prostate cancer: a prospective analysis. Urology. 2006;67:1253-6.

17. Sharifi N, Gulley JL, Dahut WL. Androgen deprivation therapy for prostate cancer. JAMA. 2005;294:238-44.

18. Tannock IF, Wit R, Berry WR, Horti J, Pluzanska A, Chi $\mathrm{KN}$, et al. Docetaxel plus prednisone or mitoxantrone plus prednisone for advanced prostate cancer. N Engl J Med. 2004;351:1502-12. 